\title{
Simulation and analysis of partial pulse remained jamming to HPRF PD radar
}

\author{
Zhiyong Sun, Chunjuan Shi ${ }^{*}$, and Hailin Tian \\ Air and Missile Defense College, Air Force Engineering University, Xi'an, China
}

\begin{abstract}
To solve the problem that the duty cycle of HPRF PD radar is not easy to use the general tow-and-pull jamming, a method of partial pulse remained jamming is proposed. Taking the transmitting signal of PD radar acquired by DRFM as an example, the jamming effect of the signal is simulated and analyzed. The results show that the jamming signal is modulated by the radar signal acquired by DRFM, the jamming signal generated has a strong correlation with the target Echo Signal, and it can effectively jam the HPRF PD radar with less power, which proves the effectiveness of the method.
\end{abstract}

Keywords: HPRF PD radar, Partial pulse remained jamming, Simulation analysis.

\section{Introduction}

The high pulse repetition rate (HPRF) PD radar is mainly used to solve the problem of velocity measuring mode and frequency in $\mathrm{x}$-band ranging from $100 \mathrm{kHz}$ to $300 \mathrm{kHz}$, high duty cycle must be used ${ }^{[1]}$. Moreover, the narrow bandwidth of HPRF PD radar makes noise jamming not very effective ${ }^{[2]}$.

To solve the problem that the duty cycle of HPRF PD radar is not easy to use the general tow-and-pull jamming, a method of pulse jamming is proposed. The PD radar transmit signal is acquired by DRFM technology, and the PD radar transmit signal is transmitted, and every $\mathrm{m}$ pulse is discarded, so the PD pulse jamming signal is produced. This kind of jamming signal keeps the coherent characteristic of the original radar signal, makes the jamming signal entering the receiver get the same processing gain as the target echo, and abandons certain pulse, compared with PD radar echo signal, the jamming signal has a great change in frequency domain, resulting in a number of frequency-shift jamming signals.

\section{Principle of DRFM}

DRFM can accurately copy the radar signal emitted by the other side, and it is a powerful means against the current advanced radar system, PD radar is no exception ${ }^{[3]}$.

\footnotetext{
* Corresponding author: $\underline{\text { scj-003@163.com }}$
} 


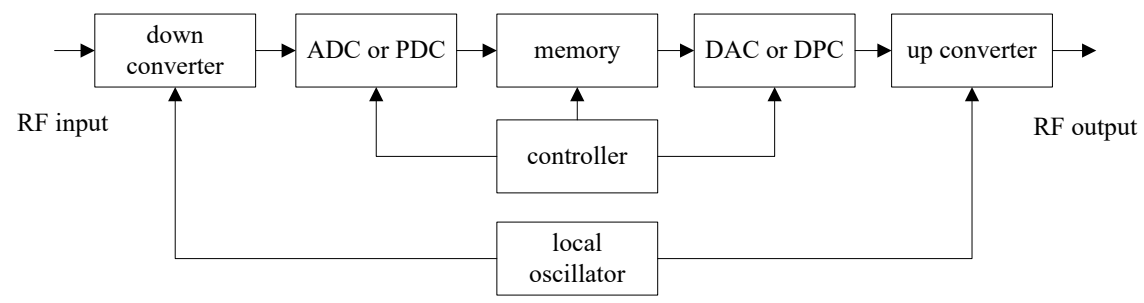

Fig. 1. Principle of DRFM.

A DRFM system typically has six basic parts: the down converter, ADC or PDC, memory, DAC or DPC, up converter, and controller, as shown in figure $1^{[4]}$. Its main working process: First, will intercept the radar signal down-conversion to if signal; then, quantitative coding, and the results will be stored in memory, the digital signal is read out from the memory at an appropriate time and decoded back to the if analog signal, which is transmitted after up-conversion to the RF signal.

\section{Analysis of frequency spectrum characteristics of PD radar}

Let the PD radar signal be

$$
s(t)=\sum_{\mathrm{n}=0}^{N} \operatorname{rec} t\left(\frac{t-n T_{r}}{\tau}\right) \cos \left(2 \pi f_{0} t\right)
$$

In the formula: $T_{r}$ is the pulse repetition period $\left(f_{r}=1 / T_{r}\right), f_{0}$ is the carrier frequency, $\tau$ is the pulse width, $N$ is the number of pulses. figure 2 is a spectrum of a HPRF PD radar signal ( $f_{r}$ is $100 \mathrm{KHz}$, duty cycle is $40 \%, N=20$ ) .

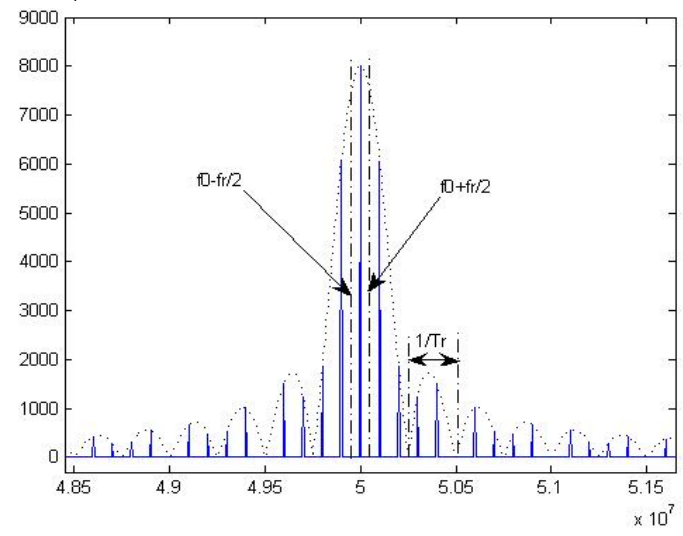

Fig. 2. Spectrum of HPRF PD radar signal.

As can be seen from figure 2, the spectrum envelope of PD Radar Echo signal is a function $S a$, whose period is the reciprocal of pulse width and the interval between the spectrum lines is $f_{r}$. In the process of signal processing of Reference PD radar, it can be seen that PD radar first passes through a single sideband filter and then enters a narrow band filter. The bandwidth of the single sideband filter is approximately equal to pulse repetition frequency $f_{r}$, and the single sideband filter is to select the echo spectrum of the central spectral line for filtering. Therefore, only when the spectrum line of jamming signal 
falls into $\left[f_{0}-\frac{f_{r}}{2}, f_{0}+\frac{f_{r}}{2}\right]$, the jamming is effective, and the more the number and the larger the amplitude, the more effective the jamming is.

\section{Principle of the method of pulse interference}

The pulse interference is generated as shown in figure 3 .

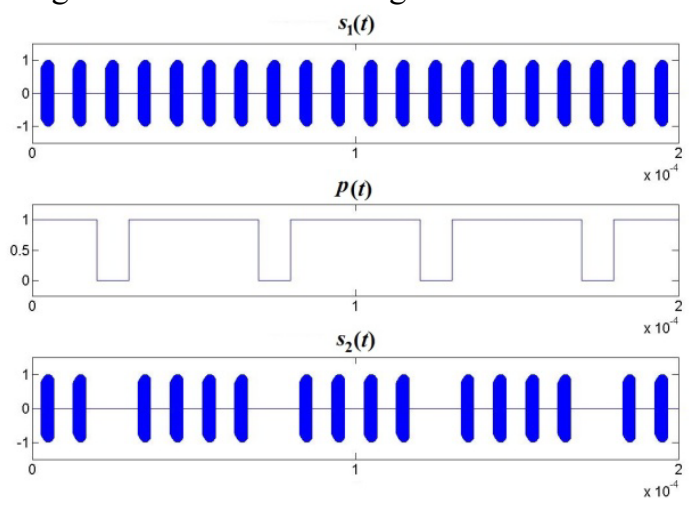

Fig. 3. Schematic diagram of pulse interference generation.

The rectangular envelope pulse train of pulse modulation is set to

$$
\mathrm{p}(t)=\operatorname{rect}\left(\frac{t}{(M-1) T_{r}}\right) * \sum_{-\infty}^{\infty} \delta\left(t-M n T_{r}\right)
$$

The spectral function is

$$
P(f)=\sum_{-\infty}^{+\infty} a_{n} \delta\left(f-n \frac{f_{r}}{M}\right)
$$

In the formula: $a_{n}=\frac{M-1}{M} \operatorname{Sa}\left(\pi n \cdot \frac{M-1}{M}\right)$.

The radar signal picked up by DRFM formula (1) is

$$
s_{1}(t)=\sum_{\mathrm{n}=0}^{N} r e c t\left(\frac{t-t r-n T_{r}}{\tau}\right) \cos \left(2 \pi f_{0}(t-t r)\right)
$$

Which, $t r$ is the Echo time-delay, carry on the round pulse modulation to the echo signal, the schematic diagram is as shown in figure 3 , may obtain the jamming signal

$$
s_{2}(t)=s_{1}(t) p(t)=\operatorname{rect}\left(\frac{t}{(M-1) T_{r}}\right) * \sum_{-\infty}^{\infty} s_{1}\left(t-M n T_{r}\right)
$$

For the convenience of the problem, the formula (3) is Fourier series, and another expression for $s_{2}(t)$ is

$$
s_{2}(t)=\frac{\mathrm{M}-1}{\mathrm{M}} \mathrm{s}_{1}(\mathrm{t})+2 \frac{\mathrm{M}-1}{\mathrm{M}} \sum_{n=1}^{\infty} \operatorname{Sa}\left(n \frac{\mathrm{M}-1}{\mathrm{M}}\right) \cos 2 \pi n \frac{f_{r} t}{M} \cdot s_{1}(t)
$$

As can be seen from equation (6), component $[(\mathrm{M}-1) / \mathrm{M}] \mathrm{s}_{1}(\mathrm{t})$ is an intercepted radar signal, with an amplitude multiplied by a factor $(M-1) / M$. 
In addition, the summation shows that the harmonics of the round pulse modulate the intercepted radar signal $s_{1}(t)$, that is, the spectrum of $s_{1}(t)$ is moved to the harmonics of $p(t)$.

The interference signal spectrum $S_{2}(f)$ is obtained by convolution operation of the intercepted signal with formula (3).

$$
S_{2}(f)=\sum_{-\infty}^{+\infty} a_{n} S_{1}\left(f-n \frac{f_{r}}{M}\right)
$$

As can be seen from the formula (7): $S_{2}(f)$ is the periodic strict extension of $S_{1}(f)$. the periodic strict extension is $\frac{f_{r}}{M}$, The amplitude weighting Coefficient is $a_{n}$.

When $\mathrm{n}=0$, the spectrum component is $\frac{M-1}{M} S_{1}(f)$, which is the same spectrum as the original radar signal.

When $n \neq 0$, the spectrum component is $\pm \frac{M-1}{M} S a\left(\frac{(M-1) n \pi}{M}\right) S_{1}\left(f \pm n \frac{f_{r}}{M}\right)$.

Because, $S_{1}\left(f \pm n \frac{f_{r}}{M}\right) \leftrightarrow s_{1}(t) e^{\mp j \frac{2 \pi n f_{r} t}{M}}$, The spectrum shape is the same as that of the radar signal, and the frequency shift of $\pm n \frac{f_{r}}{M}$, occurs as an additional frequency shift jamming signal.

\section{Simulation experiment and result analysis}

Simulation parameters: The pulse repetition period is $100 \mathrm{KHz}$, the carrier frequency is $5 \mathrm{e}^{7}$, the duty cycle is $40 \%$, the number of pulse series is $20, f_{d}$ for $5 \mathrm{KHz}$. The simulation results the interference spectra with different values of $\mathrm{M}$ respectively are shown in figure 4.

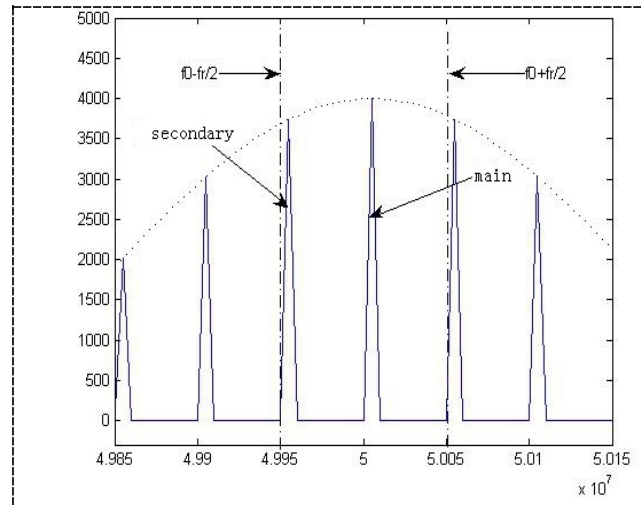

(a) $\mathrm{M}=2$

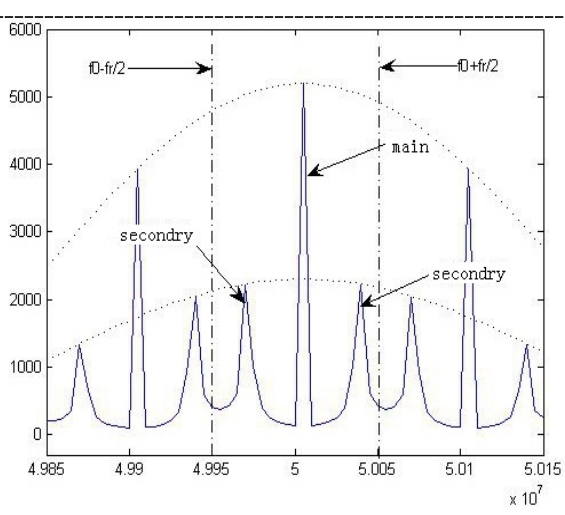

(b) $M=3$ 


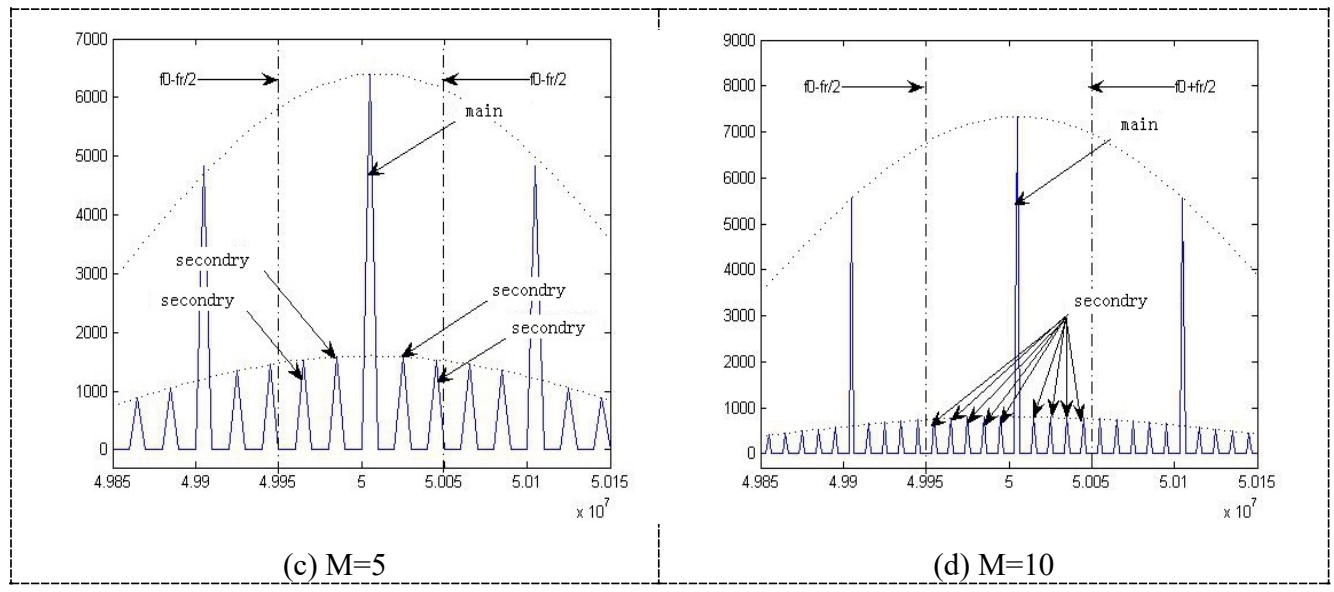

Fig. 4. Spectrum comparison chart of interference signal with different values $M$.

The following conclusions can be drawn:

(1) the number of false targets produced by jamming signal is determined by $M$, and the spectrum spacing between false targets is $\frac{f_{r}}{M}$, which is consistent with the theoretical analysis.

(2) The amplitude fluctuation of false target group is related to M. It mainly produces a strong primary false target and some weak secondary false targets. The results of theoretical analysis show that the primary false target is the $\frac{M-1}{M}$ times of the real target signal strength. The range of sub-target group is not obvious, but the range of sub-target group is quite different from that of main target group, and the bigger the $\mathrm{M}$ is, the more obvious the range is, the range of the main false target is 1 times, 2 times, 4 times and 9 times than that of the secondary false target.

Therefore, how to choose $\mathrm{M}$ is very important to the jamming effect, $\mathrm{M}$ is too small, the number of false targets is too small, $\mathrm{M}$ is too big, the range of the sub-false targets is too big relative to the main false targets group, maybe the radar receiver will treat the sub-false targets as noise, therefore, the general value of $\mathrm{M}$ is $3 \sim 5$.

\section{Conclusion}

Through the research of this paper, the main conclusions are as follows:

(1) Through the analysis of the frequency spectrum characteristics of PD radar, aiming at the characteristics of HPRF PD radar, a method of pulse jamming is put forward, and the simulation experiment is carried out.

(2) Adopting the method of round pulse jamming, by controlling the period of round pulse, the repetition period of jamming signal is changed relative to radar signal, and a number of spurious targets are produced in the bandwidth of single sideband filter of radar receiver.

(3) The radar signal obtained by DRFM and then modulated, the jamming signal generated has a strong correlation with the target echo signal, which can effectively jam the HPRF PD radar with less power, the validity of the method is verified. 


\section{References}

1. Lin Cheng Dengzhao Xie Dongxu Jiang and Yun Zhang 2017 Pulse-Doppler radar simulation research based on System Ship electronic countermeasure. vol 40 pp 3342

2. Zhiyong Sun Hong Tang and Chunjuan Shi 2012 Jamming HPRF PD radar using partial pulse remained method[J]. Modern Defence Technology vol 40 pp 115-119

3. Feng Liu and Weike Lai 2019 Study on the mechanism of speed deception jamming for airborne PD radar Electronic design engineering vol 27 pp 38-44

4. Yanqiao Gui Yanhong Wu and Daobin Yu 2018 Study on fire and command control of PD radar with multi-phase segmented modulation jamming. Fire and command control vol 43 pp 68-73 